\title{
Cerebral Ischemia in Patients on Non-Vitamin-K-Dependent Oral Anticoagulants: Through Plasma-Levels on Admission are Associated with Stroke Severity
}

Kosmas Macha로 MD; Armin Marsch ${ }^{1}$, MD; Gabriela Siedler, MD; Stefan Schwab, MD; Bernd Kallmünzer, MD;

${ }^{1}$ Authors contributed equally

Department of Neurology, University of Erlangen-Nuremberg, Erlangen, Germany

Background: Non-Vitamin-K-dependent oral anticoagulants (NOAC) are increasingly common in primary and secondary prevention of ischemic stroke. If an ischemic stroke occurs under NOAC-therapy, the severity and clinical course might be affected by the quality of anticoagulation at the time of stroke onset, but clinical data are limited.

Methods: Data from our prospective observational registry including all patients admitted with acute cerebral ischemia while taking oral anticoagulants between November 2014 and October 2017 were investigated. Functional through plasma-levels were measured in NOAC-patients using the calibrated Xaactivity (Apixaban, Rivaroxaban, Edoxaban) or the Hemoclot-essay (Dabigatran) The activity of anticoagulation was categorized into low $(<50 \mathrm{ng} / \mathrm{ml})$, intermediate $(50-100 \mathrm{ng} / \mathrm{ml})$, or high $(>100 \mathrm{ng} / \mathrm{ml})$ and its association with clinical and imaging characteristics were investigated.

Results: Of 234 patients treated with NOAC on admission, functional through plasma-levels were available in 177 subjects (75.6\%).

Plasma-levels were found to be low in 49 (27.7\%), intermediate in $41(23.2 \%)$, and high in 87 patients (49.2\%). Patient groups were similar for relevant demographic parameters. Patients with low plasma-levels had significantly higher scores on the NIHSS-scale on admission (median 8 (IQR 3-15) vs. 4 (1-11) vs. $3(0-8) ; p=0.005)$ and had a higher risk of persisting neurologic deficits or cerebral infarction on imaging (diagnosis stroke: 42 (85.7\%) vs. 31 (75.6\%) vs. 47 (54.0\%); $p<0.001)$. In addition, occlusion of a large cerebral vessel was more frequent with low plasma-levels (23 (46.9\%) vs. 9 (22.0\%) vs. 13 (14.9\%); $p<0.001)$.

Patients with large vessel occlusion (LVO) ( $n=45)$ on admission were younger (median 77.0 years (IQR 72.5-83.0) vs. 81.0 (77.0-86.0); $p=0.003$ ), had a better renal function (normal renal function on admission $68.9 \%$ vs. $49.2 \% ; p=0.023$ ), and presented earlier to the hospital than patients without large vessel occlusion (within 4.5 hours post symptom onset $66.7 \%$ vs. $43.5 \%$; $p=0.008$ / within 12 hours post symptom onset $100.0 \%$ vs. 90.9\%; $p=0.038$ ). In patients presenting with LVO through plasma-levels on admission were more often categorized low $(51.1 \%$ vs. $19.7 \% ; p<0.001)$ and less often categorized high ( $28.9 \%$ vs. $56.1 \%$; $p=0.002$ ) compared to patients without LVO. In multivariate logistic regression analyses low though plasma-level was independently associated with higher odds of LVO on admission $(3.84$ (95\% Cl 1.80-8.20); $p=0.001)$.

Conclusion: Through plasma-levels of NOAC on admission are associated with stroke severity. Low NOAC-plasma-levels on admission were independently associated with higher odds of large vessel occlusion on admission.

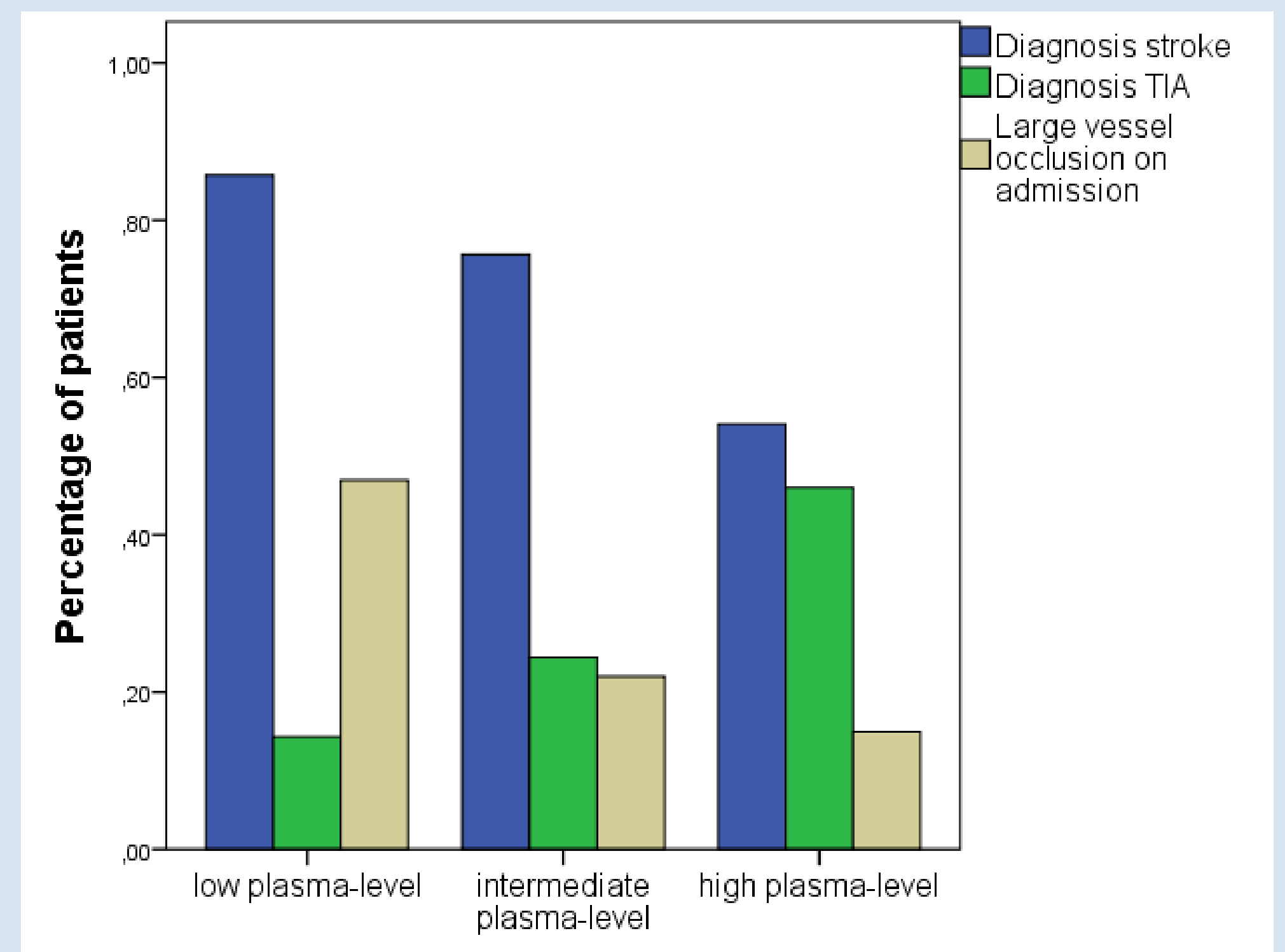

(a) (1) DV: $23(46.9 \%)$ vs. $9(22.0 \%)$ vs. $13(14.9 \%)$; $p<0.001$

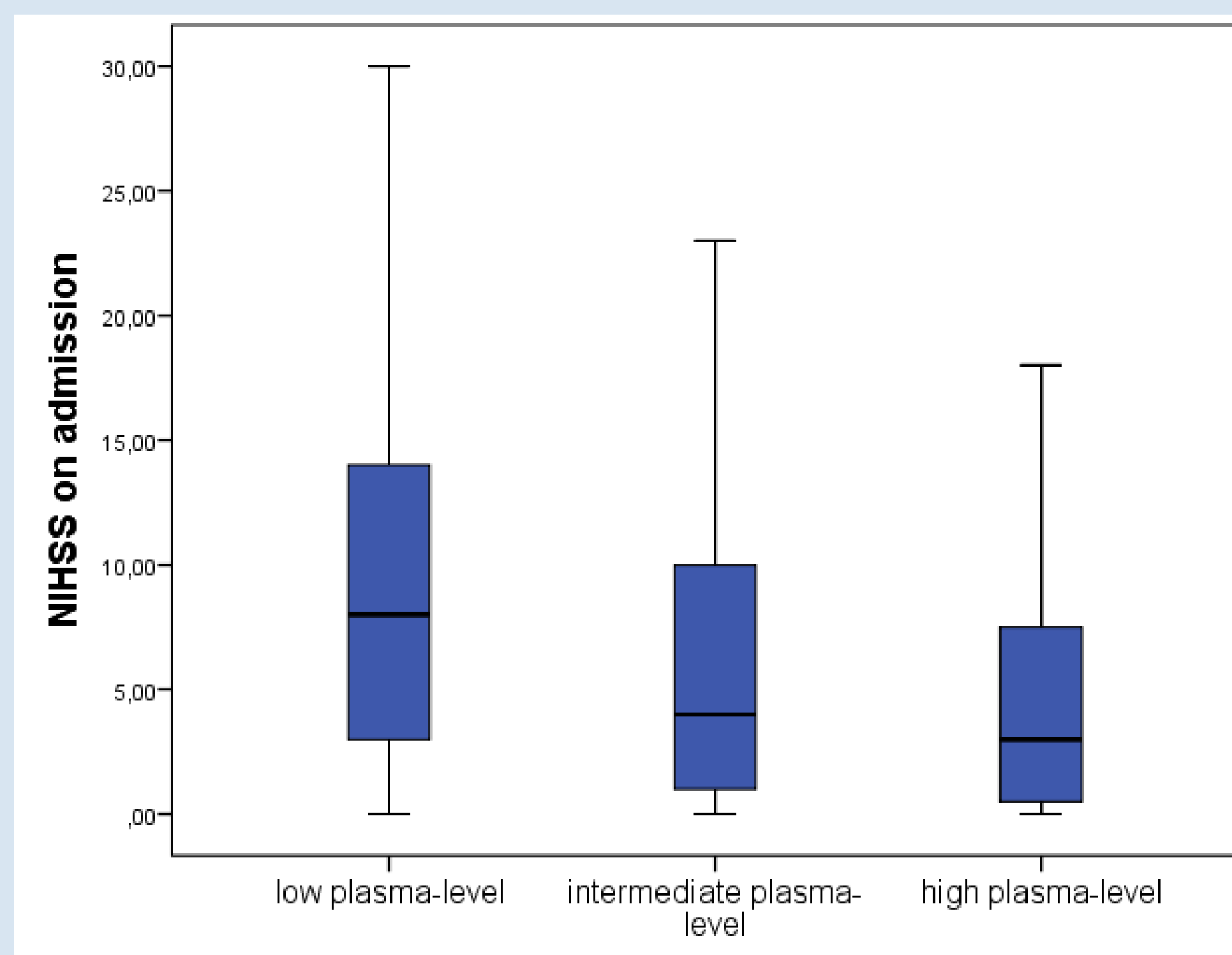

Median NIHSS on admission in patients catesorized to plasma-levels low, intermediate and high.

\begin{tabular}{|c|c|c|c|}
\hline & \begin{tabular}{|l} 
No Large Vessel Occlusion on \\
Admission \\
( $n=132)$
\end{tabular} & \begin{tabular}{|l} 
Large Vessel Occlusion on \\
Admission \\
(n=45)
\end{tabular} & p-value \\
\hline Age & $81.0(77.0-86.0)$ & $77.0(72.5-83.0)$ & 0.003 \\
\hline Median (IQR) & & & \\
\hline Male sex & $60(45.5)$ & $24(53.3)$ & 0.361 \\
\hline$n(\%)$ & & & \\
\hline Arterial hypertension & $124(93.9)$ & $42(93.3)$ & 1.000 \\
\hline Diabetes mellitus & $44(33.3)$ & $12(26.7)$ & 0.406 \\
\hline$n$ (\%) & & & \\
\hline $\begin{array}{l}\text { Hyperlipidemia } \\
\text { n (\%) }\end{array}$ & $95(72.0)$ & $29(64.4)$ & 0.341 \\
\hline Normal renal function & $65(49.2)$ & $31(68.9)$ & 0.023 \\
\hline$n(\%)$ & & & \\
\hline $\mathrm{CHA}_{2} \mathrm{DS}_{2}-\mathrm{VASC}$ & $6.0(5.0-7.0)$ & $6.0(5.0-7.0)$ & 0.175 \\
\hline Median (IQR) & & & \\
\hline NIHSS & $3.0(1.0-5.75)$ & $15.0(9.0-18.5)$ & $<0.001$ \\
\hline Median (IQR) & & & \\
\hline NOAC-dosis low & $67(50.8)$ & $\begin{array}{r}21 \text { (46.7) } \\
\text { Data missing in } 1 \text { natient }\end{array}$ & 0.728 \\
\hline IV-Thrombolysis & $3(2.3)$ & $\begin{array}{l}\text { Data missing in } 1 \text { patient } \\
\qquad 14(31.1)\end{array}$ & $<0.001$ \\
\hline$n(\%)$ & & & \\
\hline $\begin{array}{l}\text { Endovascular treatment } \\
\text { n (\%) }\end{array}$ & $0(0.0)$ & $25(55.6)$ & $<0.001$ \\
\hline $\begin{array}{l}\text { Plasma-level low } \\
\text { n (\%) }\end{array}$ & $26(19.7)$ & $23(51.1)$ & $<0.001$ \\
\hline $\begin{array}{l}\text { n (\%) } \\
\text { Plasma-level intermediate }\end{array}$ & $32(24.2)$ & $9(200)$ & 0.560 \\
\hline$n(\%)$ & & & \\
\hline $\begin{array}{l}\text { Plasma-level high } \\
\text { n (\%) }\end{array}$ & $74(56.1)$ & $13(28.9)$ & 0.002 \\
\hline Time frame $<4.5 \mathrm{~h}$ & $58(43.9)$ & $30(66.7)$ & 0.008 \\
\hline $\begin{array}{l}\mathrm{n}(\%) \\
\text { Time frame }<12 \mathrm{~h} \\
\mathrm{n}(\%)\end{array}$ & $120(90.9)$ & $45(100.0)$ & 0.038 \\
\hline
\end{tabular}

\begin{tabular}{|l|l|r|}
\hline Variable & \multicolumn{2}{l|}{ Univariable analyses } \\
\hline & OR $(95 \% \mathrm{Cl})$ & \multicolumn{1}{l|}{$\mathrm{p}$-value } \\
\hline & & \\
\hline Age & $0.94(0.91-0.98)$ & 0.006 \\
\hline Male sex & $1.37(0.70-2.70)$ & 0.362 \\
\hline $\mathrm{CHA}_{2}$ DS 2 -VASC & $0.84(0.66-1.06)$ & 0.143 \\
\hline Pre-mRS & $0.83(0.65-1.06)$ & 0.132 \\
\hline NOAC low & $0.89(0.45-1.75)$ & 0.728 \\
\hline Normal renal function & $2.28(1.11-4.68)$ & 0.024 \\
\hline Plasma-level low & $4.26(2.06-8.80)$ & $<0.001$ \\
\hline Plasma-level intermediate & $0.78(0.34-1.80)$ & 0.561 \\
\hline Plasma-level high & $0.32(0.15-0.66)$ & 0.002 \\
\hline
\end{tabular}

